\title{
Limiar e latência do reflexo acústico sob efeito de estimulação contralateral
}

\section{Threshold and latency of acoustic reflex under effect of contralateral noise}

\author{
Isabella Elias Burjato Raposo do Amaral ${ }^{1}$, Renata Mota Mamede Carvallo ${ }^{2}$
}

\begin{abstract}
RESUMO
Objetivo: Investigar o efeito inibitório da via eferente auditiva na variação do limiar e da latência do reflexo acústico ipsilateral com estimulação contralateral. Métodos: Foram avaliados 17 pacientes entre 18 e 30 anos, com audição dentro dos padrões de normalidade submetidos à pesquisa de limiar e de latência do reflexo acústico, com e sem estimulação contralateral. Resultados: Foram observadas médias de latência sem ruído contralateral para as frequiências de 500, 1000 e $2000 \mathrm{~Hz}$ respectivamente em 234,48, 214,96 e 236,71 milissegundos. Os valores de latência com ruído nas mesmas frequiências foram 230,74, 214,00 e 232,15 milissegundos. Conclusão: Houve diminuição da latência e aumento dos limiares do reflexo acústico quando apresentado estímulo supressor na orelha contralateral.
\end{abstract}

Descritores: Reflexo acústico; Tempo de reação; Vias eferentes; Estimulação acústica; Orelha média

\section{INTRODUÇÃO}

A busca de novas informações sobre o papel do sistema auditivo eferente na função auditiva periférica tem sido preocupação de vários pesquisadores ${ }^{(1-2)}$. A via eferente auditiva é descrita como de importante papel no processamento do sinal acústico em presença de ruído competitivo, na proteção contra o ruído elevado e na localização da fonte sonora $^{(3)}$. Desta forma, estudos que envolvem a avaliação da via eferente auditiva podem contribuir para o avanço do conhecimento em processamento auditivo. Pouco é conhecido sobre o papel da via eferente, no entanto muitos autores concordam que o seu efeito inibitório exerça influência no controle da informação sonora que segue em direção ao córtex auditivo. Estudos sobre via eferente requerem que ela seja ativada, de forma a permitir a análise de seu efeito inibitório. O procedimento que melhor permite a ativação da via eferente é a aplicação de um ruído supressor de faixa larga simultâneo ao estímulo ativador.

A via eferente auditiva exerce influência tanto nas condi-

Trabalho realizado no Curso de Fonoaudiologia da Faculdade de Medicina da Universidade de São Paulo - FMUSP - São Paulo (SP), Brasil.

(1) Especialização em Fonoaudiologia pela Faculdade de Medicina da Universidade de São Paulo - USP - São Paulo (SP), Brasil; Colaboradora do Laboratório de Investigação Fonoaudiológica em Audição Humana da Faculdade de Medicina da Universidade de São Paulo -USP - São Paulo (SP), Brasil.

(2) Livre Docente, Professora Associada do Curso de Fonoaudiologia da Faculdade de Medicina da Universidade de São Paulo -USP - São Paulo (SP), Brasil.

Endereço para correspondência: Isabella Elias Burjato Raposo do Amaral. R. Lopes Trovão, 09, José Menino, Santos - SP, CEP 11065-630. E-mail: isabellaburjato@yahoo.com.br

Recebido em: 13/6/2007; Aceito em: 17/12/2007 ções mecano-elétricas das células ciliadas externas da cóclea, como nos músculos da orelha média ${ }^{(2)}$. O controle eferente dos músculos da orelha média é largamente estudado por meio das medidas imitanciométricas de pesquisa de reflexos acústicos ${ }^{(4)}$. Estímulos de alto nível de intensidade sonora deflagram a contração reflexa, gerando atenuação na entrada do sinal acústico. A introdução de um segundo sinal acústico com a função de ativar a via eferente (sinal supressor) simultâneo ao estímulo ativador pode ativar mecanismos eferentes provocando mudanças nos parâmetros dos reflexos acústicos ${ }^{(5)}$.

O reflexo acústico é uma contração involuntária dos músculos da orelha média em resposta a um estímulo sonoro. O reflexo acústico pode ser investigado tanto por seu limiar (menor intensidade sonora capaz de gerar uma mudança na admitância), quanto pelo tempo de contração reflexa (ou latência do reflexo acústico). A sensibilização auditiva, fenômeno investigado na pesquisa dos reflexos acústicos, provoca a redução do limiar de reflexo acústico a partir da aplicação de um estímulo facilitador, que pode ser apresentado antes ou simultaneamente ao tom eliciador do reflexo acústico ${ }^{(6)}$.

A latência do reflexo acústico investiga a diferença de tempo entre a apresentação do sinal ativador e o surgimento do reflexo acústico propriamente dito. Estudos mostraram que a latência do reflexo acústico no ser humano varia de 150 a 200 ms para sons em níveis de 20 a $10 \mathrm{~dB}$ acima do limiar do reflexo aproximadamente ${ }^{(7-8)}$.

A pesquisa do reflexo pode ser efetuada por estimulação no lado contra ou ipsilateral à orelha avaliada. A estimulação ipsilateral conta com a apresentação do estímulo (sinal ativador) e a captação do reflexo na mesma orelha. A presença de outro sinal (estímulo supressor ou mascarante) aplicado na 
orelha contralateral permite analisar o efeito inibidor da via eferente auditiva no padrão de latência do reflexo acústico. Por meio deste procedimento é possível a análise da diferença na admitância do sistema da orelha média, entre as condições sem e com o estímulo supressor.

Estudo anterior ${ }^{(9)}$ apresentou evidências de uma via envolvendo a formação reticular. A função desta via ainda é desconhecida, mas pode estar envolvida em características complexas de reflexo acústico como efetuar a resposta antecipatória, ou seja, melhorar a resposta do reflexo acústico (redução do limiar), observada em procedimentos de sensibilização auditiva.

Os estudos de latência podem ter valor na identificação de alterações do sistema auditivo, incluindo aquelas relacionadas ao processamento auditivo, em suas funções associadas à via eferente auditiva.

O processamento auditivo é o termo utilizado para se referir à série de processos que envolvem predominantemente as estruturas do sistema nervoso central: vias auditivas e córtex ${ }^{(10)}$.

Processamento auditivo, de acordo com a American Speech and Hearing Association - $\mathrm{ASHA}^{(11)}$ refere-se à eficiência e eficácia com a qual o sistema nervoso central utiliza a informação auditiva. É o conjunto dos mecanismos e processos responsáveis pelos seguintes fenômenos comportamentais: localização e lateralização sonora; discriminação auditiva; reconhecimentos de padrões auditivos; aspectos temporais da audição, incluindo resolução temporal, mascaramento temporal, integração temporal e ordenação temporal; desempenho auditivo na presença de sinais competitivos e desempenho auditivo com sinais acústicos degradados.

As pesquisas em processamento auditivo buscam estudar os transtornos do sistema auditivo central que ocorrem nas diferentes faixas etárias.

As características mais comuns em crianças com transtorno de processamento auditivo são o déficit de compreensão da fala na presença de ruído; a desatenção; a distração; a dificuldade de comunicação; o baixo desempenho acadêmico incompatível com o nível de inteligência; memória auditiva limitada; dificuldade de identificação da idéia principal de um enunciado e baixa capacidade de interpretação de anedotas, metáforas e trocadilhos ${ }^{(3)}$.

A integridade das estruturas da via auditiva é importante para que o reflexo seja produzido com os estímulos sonoros habituais ( 75 - 85 decibel nível de audição - dBNA). Havendo lesão, o reflexo pode ocorrer apenas com sons mais intensos, ou mesmo estar completamente ausente ${ }^{(12)}$.

O sistema auditivo é organizado de forma a captar estímulos sonoros que são propagados no meio aéreo, amplificá-los e conduzi-los ao órgão de Córti, atravessando as estruturas da orelha média, e então transduzi-los em informação neural que se dirige ao córtex auditivo através das vias auditivas aferentes. Controlando o fluxo de informações aferentes, um conjunto de fibras transmite informações no sentido eferente, desde o córtex auditivo até as células ciliadas da cóclea e os músculos da orelha média ${ }^{(13)}$.

Através de estimulação por alto nível de intensidade sonora, a via ipsilateral é ativada por impulsos das células sensoriais cocleares que são transmitidos pelo nervo acústico ao núcleo coclear ventral ipsilateral. Já no reflexo contralateral, após a estimulação do nervo acústico e do núcleo coclear, os impulsos são transmitidos ao complexo olivar superior medial de onde cruzam para o núcleo motor facial contralateral, atingindo então o músculo estapédio contralateral ${ }^{(8)}$.

A latência do Reflexo Acústico é inversamente relacionada à intensidade do sinal ativador do reflexo e varia com a freqüência e com o nível do sinal ativador. As medidas da latência sofrem influência de diferentes fatores tais como: variação nas constantes de tempo definidas nos diferentes equipamentos disponíveis comercialmente e imprecisão na definição das constantes de tempo como o início do Reflexo Acústico.

A ativação da via eferente auditiva pode ser alcançada por meio da introdução de um ruído competitivo (estímulo supressor) na orelha contralateral. Ao longo dos anos, três tipos básicos de ruídos estão disponíveis para serem utilizados na avaliação audiológica: ruído complexo, ruído de faixa larga e ruído de faixa estreita ${ }^{(14)}$. O ruído de faixa estreita (narrow band) não é, na verdade, um terceiro tipo de ruído, mas é um ruído branco em faixas limitadas de freqüências. Difere do ruído branco somente na largura da faixa. Dentre os ruídos de faixa larga podem ser descritos o ruído branco ou White Noise, e o ruído rosa ou Pink Noise. O ruído branco tem sido o mais empregado em estudos de ativação da via eferente, motivo pelo qual foi selecionado para aplicação neste estudo.

Este trabalho tem como objetivo investigar o efeito inibitório da via eferente auditiva na variação do limiar e da latência do reflexo acústico ipsilateral com estimulação simultânea contralateral em três freqüências analisadas: 500 Hertz, 1000 Hertz e 2000 Hertz.

\section{MÉTODOS}

\section{Casuística}

Foram avaliados 17 pacientes com idade entre 18 e 30 anos, dos gêneros masculino e feminino, atendidos no Centro de Docência e Pesquisa da Faculdade de Medicina da Universidade de São Paulo no ano de 2002, tendo como critério de inclusão audição dentro dos padrões de normalidade. Foram todos voluntários para a pesquisa, concordando em participar após assinatura em Termo de Consentimento Livre e Esclarecido. O projeto foi aprovado pela Comissão de Pesquisa do Departamento de Fisioterapia, Fonoaudiologia e Terapia Ocupacional da Faculdade de Medicina da Universidade de São Paulo sob n 380/04.

\section{Equipamento}

Foram utilizados os seguintes equipamentos:

- Audiômetro Clínico GSI-61 - Grason Stadler® com dois canais independentes, microprocessado e calibrado dentro dos seguintes padrões: ANSI S3,6 - 1989; ANSI S3,43 1992. Equipado com fones Telephonics® TDH 50P e fones de inserção.

- Analisador de Ouvido Médio GSI-33 Grason Stadler® versão 2 , microprocessador para timpanometria e pes- 
quisa de reflexos acústicos estapedianos ipsilaterais. O equipamento está calibrado para as condições de altitude da cidade de São Paulo, segundo padrão ANSI S3,39 1987, e a instalação elétrica foi realizada com os cuidados necessários, de modo a atender as especificações técnicas do fabricante.

\section{Procedimento}

Todos os pacientes foram submetidos aos procedimentos iniciais para verificação dos critérios de inclusão: meatoscopia e audiometria tonal limiar por via aérea de 250 a 8000 Hertz, limiar de recepção de fala (LRF) e índice percentual de reconhecimento de fala (IPRF). A seguir, foram submetidos a imitanciometria, sendo realizada timpanometria e investigação do limiar do Reflexo Acústico.

Posteriormente, foi realizado o estudo da latência do reflexo acústico ipsilateral. Para tanto foi utilizado protocolo especial do equipamento GSI 33 - versão 2, para medidas pontuais da duração do reflexo acústico - ARLT (acoustic reflex latency test). As medidas de latência foram tomadas por meio de estímulos com duração a 5 milissegundos e em nível de intensidade de 10 dBNS (decibel nível de sensação) acima do valor obtido no reflexo e registradas por um espaço de tempo de 1000 milissegundos. Foram estudadas as freqüências de 500, 1000 e 2000 Hertz para ambas as orelhas.

O equipamento apresentou duas sequiências de estímulos para a análise da latência, cujos resultados foram mediados e digitalmente apresentados na tela.

As medidas de latência envolvem o tempo total de contração do músculo estapediano durante o reflexo acústico, sendo aproximado, neste equipamento, pelo valor descrito na condição de latência terminal, ou seja, $10 \%$ off.

O procedimento aplicado neste estudo executa a análise dos tempos parciais da contração, tendo sido selecionado o intervalo descrito para a latência terminal (10\% off), que se refere ao intervalo de tempo entre o final do estímulo e o ponto em que a curva do reflexo retorna a $10 \%$ de sua amplitude máxima. O modelo de registro gráfico dos tempos de latência do reflexo acústico é apresentado na Figura 1.

A análise estatística utilizou o Teste de Igualdade de Duas Médias (Teste T), adotado quando as variâncias populacionais são desconhecidas, porém admitidas como iguais, independentes e normais. Foi aceito o nível de significância de 5\% para valor de $\mathrm{p}$.

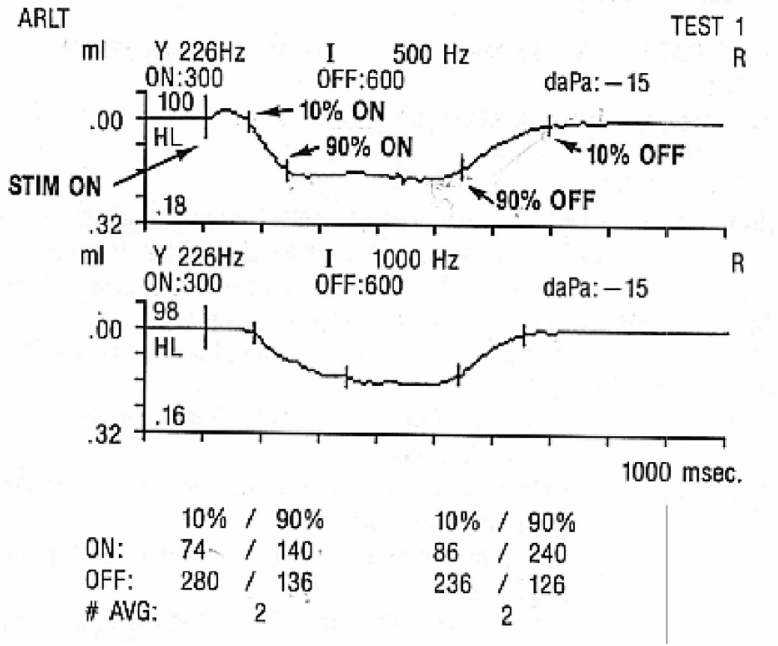

Figura 1. Registro gráfico da pesquisa da latência do reflexo acústico coletado na modalidade ipsilateral, com estímulo de $500 \mathrm{~Hz}$

\section{RESULTADOS}

As Tabelas 1 e 2 mostram os resultados das comparações entre as orelhas direita e esquerda, dos reflexos acústicos e da latência obtidos sem e com ruído contralateral.

Não existe diferença estatisticamente significante entre as orelhas nem para o Limiar do Reflexo Acústico nem para a Latência, em nenhuma das frequiências analisadas.

Desta maneira, foram reunidos os valores das orelhas para as comparações entre gêneros e entre as condições com/sem ruído, apresentadas nas Tabelas 3 e 4.

Não é estatisticamente significante a diferença existente entre os gêneros tanto no Limiar do Reflexo Acústico quanto na Latência em qualquer uma das freqüências, ou seja, os homens e as mulheres se comportam igualmente em todos os casos.

A seguir, a comparação entre as condições sem e com ruído, tanto no reflexo quanto na latência, será apresentada respectivamente, nas Tabelas 5 e 6 .

Nota-se que tanto para o Limiar do Reflexo Acústico, como para as medidas de Latência não existe diferença estatisticamente significante entre as condições sem e com ruído, em quaisquer das freqüências analisadas.

Vale observar que na pesquisa do Limiar do Reflexo Acústico, a média dos resultados sem ruído foi sempre inferior ao dos resultados com ruído (Tabela 5). Essa tendência de resultados ocorre diferentemente na pesquisa da Latência dos Reflexos

Tabela 1. Reflexos Acústicos Ipsilaterais (dBNA) sem e com o ruído contralateral, comparação entre orelhas direita e esquerda

\begin{tabular}{|c|c|c|c|c|c|c|c|c|c|c|c|c|}
\hline \multirow{3}{*}{ Orelhas } & \multicolumn{6}{|c|}{ Reflexo Acústico Sem Ruído } & \multicolumn{6}{|c|}{ Reflexo Acústico Com Ruído } \\
\hline & \multicolumn{2}{|c|}{$500 \mathrm{~Hz}$} & \multicolumn{2}{|c|}{$1000 \mathrm{~Hz}$} & \multicolumn{2}{|c|}{$2000 \mathrm{~Hz}$} & \multicolumn{2}{|c|}{$500 \mathrm{~Hz}$} & \multicolumn{2}{|c|}{$1000 \mathrm{~Hz}$} & \multicolumn{2}{|c|}{$2000 \mathrm{~Hz}$} \\
\hline & OD & OE & OD & OE & OD & OE & OD & OE & OD & OE & OD & OE \\
\hline Média & 85,4 & 88,6 & 84,9 & 84,7 & 86,0 & 87,3 & 86,5 & 88,5 & 85,1 & 86,4 & 85,9 & 88,0 \\
\hline $\begin{array}{l}\text { Desvio } \\
\text { Padrão }\end{array}$ & 6,7 & 5,5 & 6,1 & 6,7 & 6,0 & 6,4 & 4,8 & 5,6 & 5,6 & 5,8 & 5,9 & 6,2 \\
\hline Tamanho & 17 & 17 & 17 & 17 & 17 & 17 & 17 & 17 & 17 & 17 & 17 & 17 \\
\hline p-valor & \multicolumn{2}{|c|}{0,102} & \multicolumn{2}{|c|}{0,903} & \multicolumn{2}{|c|}{0,517} & \multicolumn{2}{|c|}{0,191} & \multicolumn{2}{|c|}{0,438} & \multicolumn{2}{|c|}{0,257} \\
\hline
\end{tabular}

Legenda: $\mathrm{Hz}=\mathrm{Hertz} ; \mathrm{OD}=$ Orelha Direita; $\mathrm{OE}=$ Orelha Esquerda 
Tabela 2. Latência do Reflexo Acústico Ipsilateral (dBNA) sem e com o ruído contralateral, comparação entre orelhas direita e esquerda

\begin{tabular}{|c|c|c|c|c|c|c|c|c|c|c|c|c|}
\hline \multirow{3}{*}{ Orelhas } & \multicolumn{6}{|c|}{ Latência - 10\% Off Sem Ruído } & \multicolumn{6}{|c|}{ Latência - 10\% Off Com Ruído } \\
\hline & \multicolumn{2}{|c|}{$500 \mathrm{~Hz}$} & \multicolumn{2}{|c|}{$1000 \mathrm{~Hz}$} & \multicolumn{2}{|c|}{$2000 \mathrm{~Hz}$} & \multicolumn{2}{|c|}{$500 \mathrm{~Hz}$} & \multicolumn{2}{|c|}{$1000 \mathrm{~Hz}$} & \multicolumn{2}{|c|}{$2000 \mathrm{~Hz}$} \\
\hline & OD & OE & OD & OE & OD & OE & OD & OE & OD & OE & OD & OE \\
\hline Média & $23,8,8$ & 230,5 & 219,5 & 209,3 & 253,2 & 224,4 & 229,1 & 232,3 & 222,4 & 206,1 & 237,1 & 228,5 \\
\hline $\begin{array}{l}\text { Desvio } \\
\text { Padrão }\end{array}$ & 51,2 & 53,5 & 55,9 & 36,3 & 88,3 & 59,9 & 55,4 & 70,1 & 44,8 & 32,7 & 51,7 & 73,4 \\
\hline Tamanho & 12 & 13 & 15 & 12 & 12 & 16 & 13 & 14 & 14 & 15 & 11 & 15 \\
\hline p-valor & \multicolumn{2}{|c|}{0,693} & \multicolumn{2}{|c|}{0,575} & \multicolumn{2}{|c|}{0,343} & \multicolumn{2}{|c|}{0,896} & \multicolumn{2}{|c|}{0,277} & \multicolumn{2}{|c|}{0,731} \\
\hline
\end{tabular}

Legenda: $\mathrm{Hz}=$ Hertz; OD = Orelha Direita; $\mathrm{OE}=$ Orelha Esquerda

Tabela 3. Reflexos Acústicos Ipsilaterais (dBNA) sem e com o ruído contralateral, comparação entre gêneros masculino e feminino

\begin{tabular}{|c|c|c|c|c|c|c|c|c|c|c|c|c|}
\hline \multirow{3}{*}{ Gêneros } & \multicolumn{6}{|c|}{ Reflexo Acústico Sem Ruído } & \multicolumn{6}{|c|}{ Reflexo Acústico Com Ruído } \\
\hline & \multicolumn{2}{|c|}{$500 \mathrm{~Hz}$} & \multicolumn{2}{|c|}{$1000 \mathrm{~Hz}$} & \multicolumn{2}{|c|}{$2000 \mathrm{~Hz}$} & \multicolumn{2}{|c|}{$500 \mathrm{~Hz}$} & \multicolumn{2}{|c|}{$1000 \mathrm{~Hz}$} & \multicolumn{2}{|c|}{$2000 \mathrm{~Hz}$} \\
\hline & OD & OE & OD & OE & OD & OE & OD & OE & OD & OE & OD & OE \\
\hline Média & 85,5 & 89,1 & 83,9 & 86,1 & 86,9 & 86,3 & 86,4 & 89,0 & 85,1 & 86,6 & 86,8 & 87,1 \\
\hline $\begin{array}{l}\text { Desvio } \\
\text { Padrão }\end{array}$ & 7,3 & 3,6 & 7,6 & 3,5 & 7,4 & 4,6 & 5,6 & 4,4 & 6,5 & 4,3 & 6,9 & 4,8 \\
\hline Tamanho & 20 & 14 & 20 & 14 & 20 & 14 & 20 & 14 & 20 & 14 & 20 & 14 \\
\hline p-valor & \multicolumn{2}{|c|}{0,063} & \multicolumn{2}{|c|}{0,261} & \multicolumn{2}{|c|}{0,757} & \multicolumn{2}{|c|}{0,138} & \multicolumn{2}{|c|}{0,433} & \multicolumn{2}{|c|}{0,865} \\
\hline
\end{tabular}

Legenda: $\mathrm{Hz}=\mathrm{Hertz} ; \mathrm{OD}=$ Orelha Direita; $\mathrm{OE}=$ Orelha Esquerda

Tabela 4. Latência do Reflexo Acústico Ipsilateral (dBNA) sem e com o ruído contralateral, comparação entre gêneros masculino e feminino

\begin{tabular}{|c|c|c|c|c|c|c|c|c|c|c|c|c|}
\hline \multirow{3}{*}{ Gêneros } & \multicolumn{6}{|c|}{ Latência - 10\% Off Sem Ruído } & \multicolumn{6}{|c|}{ Latência - 10\% Off Com Ruído } \\
\hline & \multicolumn{2}{|c|}{$500 \mathrm{~Hz}$} & \multicolumn{2}{|c|}{$1000 \mathrm{~Hz}$} & \multicolumn{2}{|c|}{$2000 \mathrm{~Hz}$} & \multicolumn{2}{|c|}{$500 \mathrm{~Hz}$} & \multicolumn{2}{|c|}{$1000 \mathrm{~Hz}$} & \multicolumn{2}{|c|}{$2000 \mathrm{~Hz}$} \\
\hline & OD & $\mathrm{OE}$ & OD & $\mathrm{OE}$ & OD & OE & OD & $\mathrm{OE}$ & OD & $\mathrm{OE}$ & OD & $\mathrm{OE}$ \\
\hline Média & 243,7 & 224,5 & 217,8 & 210,9 & 245,8 & 224,7 & 241,3 & 215,5 & 215,6 & 212,0 & 240,7 & 220,5 \\
\hline $\begin{array}{l}\text { Desvio } \\
\text { Padrão }\end{array}$ & \multicolumn{11}{|c|}{ Padrão } & 48,8 \\
\hline Tamanho & 13 & 12 & 16 & 11 & 16 & 12 & 16 & 11 & 16 & 13 & 15 & 11 \\
\hline $\mathrm{p}$-valor & \multicolumn{2}{|c|}{0,355} & \multicolumn{2}{|c|}{0.724} & \multicolumn{2}{|c|}{0,443} & \multicolumn{2}{|c|}{0,268} & \multicolumn{2}{|c|}{0,806} & \multicolumn{2}{|c|}{0,412} \\
\hline
\end{tabular}

Legenda: $\mathrm{Hz}=$ Hertz; OD = Orelha Direita; OE = Orelha Esquerda

Tabela 5. Limiar de Reflexos Acústicos Ipsilaterais (dBNA), comparação entre as condições sem e com ruído contralateral

\begin{tabular}{|c|c|c|c|c|c|c|}
\hline \multirow{3}{*}{ Sem Ruído vs. Com Ruído } & \multicolumn{6}{|c|}{ Limiar de Reflexo Acústico } \\
\hline & \multicolumn{2}{|c|}{$500 \mathrm{~Hz}$} & \multicolumn{2}{|c|}{$1000 \mathrm{~Hz}$} & \multicolumn{2}{|c|}{$2000 \mathrm{~Hz}$} \\
\hline & Sem Ruído & Com ruído & Sem Ruído & Com ruído & Sem Ruído & Com ruído \\
\hline Média & 87,00 & 87,47 & 84,82 & 85,71 & 86,65 & 86,95 \\
\hline Desvio Padrão & 6,21 & 5,22 & 6,31 & 5,68 & 6,14 & 6,06 \\
\hline Tamanho & 34 & 34 & 34 & 34 & 34 & 34 \\
\hline $\mathrm{p}$-valor & \multicolumn{2}{|c|}{0,339} & \multicolumn{2}{|c|}{0,071} & \multicolumn{2}{|c|}{0,432} \\
\hline
\end{tabular}

Legenda: $\mathrm{Hz}=$ Hertz; OD = Orelha Direita; $\mathrm{OE}=$ Orelha Esquerda

Acústicos, onde a média dos reflexos sem ruído é sempre maior do que a média dos resultados com ruído (Tabela 6).

\section{DISCUSSÃO}

Em nosso estudo, a aplicação simultânea de ruído branco contralateral gerou aumento dos limiares de reflexos acústicos nas freqüências de 500 Hertz, 1000 Hertz e 2000 Hertz. Não foi observado efeito da variável lado de orelha (Tabelas 1 e 2), nem da variável gênero (Tabelas 3 e 4) nas medidas pesquisadas. Apesar de tais resultados não apresentarem diferença significante (Tabelas 5 e 6) concordam com os resultados de estudo realizado anteriormente ${ }^{(5)}$, que mostram um aumento nos limiares de reflexos acústicos, para freqüências de 1000 
Tabela 6. Latência do Reflexo Acústico Ipsilateral (dBNA), comparação entre as condições sem e com ruído contralateral

\begin{tabular}{lcccccc}
\hline & & \multicolumn{3}{c}{ Latência - $10 \%$ Off } \\
Sem Ruído vs. Com Ruído & \multicolumn{2}{c}{$500 \mathrm{~Hz}$} & \multicolumn{2}{c}{$1000 \mathrm{~Hz}$} & \multicolumn{2}{c}{$2000 \mathrm{~Hz}$} \\
& Sem Ruído & Com ruído & Sem Ruído & Com ruído & Sem Ruído & Com ruído \\
\hline Média & 234,48 & 230,74 & 214,96 & 214,00 & 236,71 & 232,15 \\
Desvio Padrão & 51,48 & 62,27 & 47,59 & 39,17 & 73,36 & 64,09 \\
Tamanho & 25 & 27 & 27 & 29 & 28 & 0,808 \\
p-valor & & 0,814 & & 0,935 & 26 \\
\hline
\end{tabular}

Legenda: $\mathrm{Hz}=\mathrm{Hertz} ; \mathrm{OD}=$ Orelha Direita; $\mathrm{OE}=$ Orelha Esquerda

e $2000 \mathrm{~Hz}$, em presença de ruído contralateral.

A elevação do limiar do reflexo, com ruído, pode ser decorrente da ação da via eferente olivococlear, podendo ser atribuído a um ou mais fatores: pela possibilidade do reflexo acústico estapediano ser eliciado por efeito do ruído branco aplicado contralateralmente; pela ação inibitória da via eferente auditiva olivococlear ou ainda, pela ação de outros sistemas e mecanismos desconhecidos ${ }^{(5)}$.

Neste estudo, para assegurar a não interferência do ruído contralateral sobre o limiar de reflexos acústicos, o nível de intensidade do ruído foi mantido em 30 decibel nível de sensação (dBNS), nível este insuficiente para desencadear reflexos acústicos em indivíduos com audição normal. Além disso, os estímulos por ruído branco foram apresentados por meio de fone de inserção, condição que reduz a possibilidade de cruzamento interaural, devido ao baixo nível de intensidade de ruído branco aplicado.

A ação inibitória da via eferente olivococlear, quando ativada por estimulação contralateral foi investigada neste estudo, tendo sido possível observar que o limiar de reflexos acústicos sofreu a influência da presença de ruído contralateral. Essa constatação ficou evidenciada tanto para o limiar, quanto para a latência do reflexo acústico, com um aumento do limiar de resposta e diminuição da latência quando se aplicou o ruído contralateral. Estudo anterior ${ }^{(7)}$ mostra o efeito da aplicação de estimulação por ruído branco na latência do reflexo acústico em indivíduos com alteração de processamento auditivo.

Este efeito inibitório tem sido associado ao controle eferente do sistema auditivo, e foi também descrito em estudos sobre o efeito inibitório da via eferente sobre as respostas de emissões otoacústicas em diferentes faixas etárias ${ }^{(15-17)}$
A ativação da via eferente olivococlear gera modificações inibitórias no sistema auditivo, que podem ser inferidas pela variação do nível de resposta das emissões otoacústicas (com e sem aplicação de ruído simultâneo). Esta variação no nível de resposta é resultante de efeito inibitório sobre a atividade das células ciliadas externas (CCE). Este efeito isoladamente não justificaria a ocorrência de efeito inibitório sobre o reflexo acústico, porém o efeito inibitório eferente poderia ter um efeito global na membrana basilar ${ }^{(18-20)}$, modificando as propriedades eletro-mecânicas da cóclea, contribuindo para uma redução nos disparos elétricos das fibras auditivas ${ }^{(21)}$. Esta redução na atividade da via auditiva gera, por sua vez, uma diminuição na amplitude de reflexo acústico. Isso pode sustentar a hipótese de que o sistema auditivo eferente, por meio de sua ação na orelha média, possa proteger a cóclea de estimulação acústica em alta intensidade ${ }^{(22)}$.

Futuros trabalhos envolvendo o estudo do efeito da ativação da via eferente auditiva nas medidas de limiar e latência do reflexo acústico, permitirão o aprimoramento do conhecimento da função do reflexo acústico.

\section{CONCLUSÃO}

Os resultados do presente estudo permitem concluir que a aplicação de ruído contralateral tem influência nas medidas de reflexos acústicos, com caráter de:

- discreto aumento do limiar de reflexo para estímulos de $500 \mathrm{~Hz}, 1000 \mathrm{~Hz}$ e $2000 \mathrm{~Hz}$;

- discreta diminuição da latência do reflexo acústico para estímulos de $500 \mathrm{~Hz}, 1000 \mathrm{~Hz}$ e $2000 \mathrm{~Hz}$.

\begin{abstract}
Purpose: To investigate the inhibitory effect of the efferent auditory path in the variation of the threshold and the latency of ipsilateral acoustic reflex with contralateral stimulation. Methods: Seventeen male and female patients, with ages between 18 and 30 years and with average normal hearing, were evaluated. After verification of inclusion criteria, the subjects were submitted to acoustic reflex threshold and latency testings, with and without contralateral masking. Results: The latency average rates without contralateral noise at the frequencies $500 \mathrm{~Hz}, 1000 \mathrm{~Hz}$ and $2000 \mathrm{~Hz}$ were, respectively, 234,48, 214,96 and 236,71 milliseconds. The latency rates with noise at the same frequencies were 230,74, 214,00 and 232,15 milliseconds. Conclusion: The results showed latency decrease and increase on the acoustic reflex thresholds with contralateral white noise suppressor stimulus.
\end{abstract}

Keywords: Reflex, acoustic; Reaction time; Afferent pathways; Acoustic stimulation; Ear, middle 


\section{REFERÊNCIAS}

1. May BJ, Budelis J, Niparko JK. Behavioral studies of the olivocochlear efferent system. Arch Otolaryngol Head Neck Surg. 2004;130(5):6604.

2. Quaranta N, Scaringi A, Nahum S, Quaranta A. Effects of efferent acoustic reflex activation on psychoacoustical tuning curves in humans. Acta Oto-Laryngologica. 2005;125(5):520- 3.

3. Chermak GD, Musiek FE. Central Auditory processing disorders: New perspectives. San Diego: Singular Publishing Group; 1997.

4. Borg E. Acoustic Middle ear reflexes: a sensory-control system. Acta Oto-Laryngologica Suppl. 1972;304: 5-33.

5. Kumar A, Barman A. Effect of efferent-induced changes on acoustical reflex. Int J Audiol. 2002;41(2):144-7.

6. Soares JC, Carvallo RMM. Redução do limiar de reflexo acústico em neonatos sem risco auditivo. Rev Bras Otorrinolaringol. 2006; 72(1):49-54.

7. Linares AE, Carvallo RMM. Latência do Reflexo Acústico em crianças com alteração do processamento auditivo. Arq.Otorrinolaringol. 2004;8(1):11-8.

8. Counter AS, Borg E. Acoustic middle ear muscle reflex protection against magnetic coil impulse noise. Acta Otolaryngol. 1993;113(4):483-8.

9. Borg E. on the neuronal organization of the acoustic middle ear reflex. a physiological and anatomical study. Brain Res.1973;49(1):101-23.

10. Schochat E, Musiek FE. Maturation of outcomes of behavioral and electrophysiologic tests of central auditory function. J Commun Disord. 2006;39(1):78-92.

11. ASHA: American Speech Language Hearing Association (2005) [Internet]. Central Auditory Processing Disorders - The Role of the Audiologist (Position statement). [cited 2007 Dec 12]. Available at http://www.asha.org/members/deskref-journals/deskref/default.

12. Castagno LA. Predição do limiar auditivo através do reflexo estapédico: uma nova fórmula de regressão linear. F Méd. 1990;101(1):45-52.
13. Carvallo RM. Medidas eletroacústicas da audição. In: Carvallo RM, editor. Fonoaudiologia: informação para formação. Rio de Janeiro: Guanabara Koogan; 2003.

14. Sanders JW, Hall JW. Mascaramento clínico. In: Musiek FE, Rintelmann WF, editor. Perspectivas atuais em avaliação auditiva. São Paulo: Manole; 2001. p. 63-84.

15. Durante, AS Carvallo, RM. Contralateral suppression of otoacoustic emissions in neonates. Int J Audiol. 2002;41(4):211- 5.

16. Sanches SG, Carvallo RM. Contralateral suppression of transient evoked otoacoustic emissions in children with auditory processing disorder. Audiol Neurootol. 2006;11(6):366-72.

17. Carvallo RMM. Audição em alta freqüência: repercussões no reconhecimento de fala no ruído e nas emissões otoacústicas [tese livredocência]. São Paulo: Faculdade de Medicina da Universidade de São Paulo; 2002.

18. Dolan DF, Nuttal AL. Basilar membrane movement evoked by sound is altered by electrical stimulation of the crossed olivocochlear bundle. Assoc Res Otolaryngol Abstr. 1994;17:89.

19. Montain DC. Changes in endolymphatic potential and crossed olivocochlear bundle stimulation alter cochlear mechanics. Science. 1980;210(4465):71-2.

20. Collet L, Kemp DT, Veuillet E, Duclaux R, Moulin A, Morgon A. Effect of contralateral auditory stimuli on active cochlear micro mechanical properties in human subjects. Hear Res. 1990;43(2-3):251-61.

21. Guinan JJ Jr, Gifford ML. Effects of electrical stimulation of efferent olivocochlear neurons on cat auditory-nerve fibers. III. Tuning curves and thresholds at CF. Hear Res. 1988;37(1):29-45.

22. Rajan R. Protected functions of the efferent pathways to the mammalian cochlea: a review. In: Dancer AL, Henderson D, Salvi RJ, editors. Noise induced hearing loss. St Louis: Mosby Yearbook; 1992. p. 429-44. 